\title{
Historical literature review and molecular analysis of malaria drug resistance markers of Plasmodium falciparum field-isolates from Sudan.
}

Nouh S. Mohamed (D Nouh_saad@outlook.com )

NURI https://orcid.org/0000-0001-6843-3361

Hanadi Abdelbagi

Ahfad University for Women School of Pharmacy

Hussam A. Osman

Ahfad University for Women School of Pharmacy

Abdallah E. Ahmed

Nile College

Alaa M. Yousif

Ahfad University for Women School of Pharmacy

Yusraa B. Edris

Ahfad University for Women School of Pharmacy

Eman Y. Osman

Ahfad University for Women School of Pharmacy

Aahd R. Elsadig

National University

Emmanuel E. Siddig

Mycetoma Research Center

Madinna Mustafa

Nile University

Ammar A. Mohammed

Sudan Federal Ministry of Health

\section{Yousif Ali}

Sudan Federal Ministry of Health

Maha M. Osman

Alfarrabi College for Science and Technology

Mohamed S. Ali

Al-Neelain University Faculty of Medicine

Rihab A. Omer

Universitat Leipzig

Ayman Ahmed 
Institute of Endemic Diseases

Carol H. Sibley

University of Washington

Research

Keywords: Plasmodium falciparum, Molecular Markers, Multi drug resistance, Sudan.

Posted Date: February 26th, 2020

DOl: https://doi.org/10.21203/rs.2.24565/v1

License: (c) (1) This work is licensed under a Creative Commons Attribution 4.0 International License. Read Full License 


\section{Abstract}

Background Malaria infection is still known to be a worldwide public health problem, especially in tropical and sub-tropical African countries like Sudan. The fight against malaria is still taking place due to many factors. One of these factors is the presence of Plasmodium falciparum drug resistant parasites. This study is aiming at studying the P. falciparum drug resistance markers and analyzing the historical literature on these markers in Sudan. Methods A descriptive cross-sectional healthcare-centers based study conducted in Khartoum state between December 2017 and July 2018. Febrile patients diagnosed with P. falciparum malaria infection were recruited. Two $\mathrm{ml}$ blood samples were collected prior to start treatment. Genotyping of the specific point mutations in the P. falciparum genome was done using Sanger sequencing method for the Pfcrt, Pfmdr-1, Pfdhfr, and Pfdhps genes. Data deposited by the worldwide antimalarial resistance network was consulted and the molecular markers previously reported from Sudan were identified, collected, and analyzed to compare between past and present frequency of malaria drug resistance mutations. One-way ANOVA test was used to calculate the least significance of frequency distribution in the molecular markers collected from the previous reports from Sudan in comparison to this study. Pearson correlation was used to investigate the association between the different drug resistance markers. Results Drug molecular markers analysis was successfully done on the 20 P. falciparum isolates. the Pfcrt K76 showed the highest frequency; 16 (80\%). Pfcrt 76T was 4 (20\%). For the Pfmdr-1 marker, 9 (45\%) isolates were carrying the N86 allele and $11(55 \%)$ were $86 \mathrm{Y}$ allele. While the Y184F of the Pfmdr-1 showed higher frequency of 184F compared to Y184; 16 (80\%) and 4 (20\%), respectively. Concerning the double Pfmdr-1 haplotype, NY haplotype was 2 (10\%), NF was 7 (35\%), YF was 9 (45\%), and YY was 2 (10\%). In the Pfdhfr , 51I allele showed higher frequency compared to N51; 18 (90\%) and $2(10 \%)$, respectively. Whereas for C59R, C59 was 18 (90\%), and 59R was $2(10 \%)$. For S108N, $18(90 \%)$ for $108 \mathrm{~N}$ and $2(10 \%)$ for S108. The triplet haplotype ICN of the Pfdhfr; was the most frequent haplotype; 16 (80\%). Concerning the Pfdhps , all the 20 (100\%) isolates were carrying the mutant alleles; $437 \mathrm{G}$ and $540 \mathrm{E}$. the Pfdhps haplotype present was the double GE haplotype only. No statistically significant correlation was found for the Pfcrt, Pfmdr-1, Pfdhfr, and Pfdhps. Historical reports on P. falciparum multidrug resistant collected from 1989 to 2016 showed extreme fluctuation. High prevalence of Pfcrt 76T allele was observed in Khartoum throughout all years of previous studies, while in Gedaref Pfcrt 76T showing increased prevalence each year. All studied genes were showing increase prevalence of the mutant alleles and reduction of the wildtype alleles. In this study, the GE mutant haplotype was prevalent in all the studied samples. Frequency distribution of the Pfcrt K76T and Pfmdr-1 N86Y alleles, Pfmdr-1 ; N86Y and Y184F, Pfdhfr ; N51I and S108N, and Pfdhps ; A437G and K540E double haplotypes was significantly different across the whole years in Sudan. Conclusion This study describes the distribution of P. falciparum multidrug resistance markers throughout Sudan providing a solid baseline data of the status of these markers which could be very useful for the malaria control program not only for establishing surveillance system that monitor the change in and/or the emergence of malaria drug resistance but it will also offer a guidance for the evidence-base decision-making regarding the treatment protocol national and regional wise. 


\section{Background}

Malaria infection is still known to be a worldwide public health problem, especially in tropical and subtropical African countries [1]. Based on the WHO estimations in 2019, malaria infections reached 228 million cases with 405 thousand related-deaths [1]. In Sudan, anti-malarial resistance among Plasmodium falciparum had emerged since 1955 investigated by Abbot, who firstly reported the phenomenon of drug-resistant in Wad Madani when using Amodiaquine-hydrochloride (AQ) and Pyrimethamine $(P)$ to treat $P$. falciparum infections [2]. During the late 70 s of the last century, high proportions of drug resistance were reported when Chloroquine (CQ) was introduced as the first-line treatment [3]. In 1991, Babiker and his colleagues investigated the drug resistance against CQ, Mefloquine (MQ) and P. They reported that drug response varieties where some isolates in in-vitro testing were found to be resistant for both $C Q$ and $P$, while others were resistant to either $C Q$ or $P$, whereas, no resistance against MQ has been reported [4]. Also, a similar case resistance against $P$ was published by Ibrahim et al., 1991 in Sennar state-Sudan [5]. Other studies reported malaria drug resistance throughout the country [6-10]. However, by 2004 the National Malaria Programme (NMP) changed the malaria management protocol and shifted from CQ to the artemisinin-based combination treatment (ACTs). The combination of Artesunate (AS) and Sulfadoxine-Pyrimethamine (SP) was then adopted as the first-line treatment against uncomplicated falciparum malaria, and artemether-lumefantrine (AL) was considered as a second-line treatment [11]. Therapeutic Efficacy Studies (TES) were conducted to monitor and detect the emergence of drug-resistant malaria parasites. Another approach for the early detection of the emergence of drug resistance was implemented by using molecular markers in order to investigate the efficacy of treatments in vitro. Once the deployment of AS+SP as a first-line treatment for $P$. falciparum malaria, TESs were conducted to evaluate SP in the form of monotherapy or combined with other anti-malarial drugs. These studies reported different efficacies of treatment and failure of SP monotherapy [12-16]. Between 2010 and 2015, Ahmed et al. conducted a study to evaluate the efficacy of AS+SP and AL and AL showed high efficacy with declining efficacy of AS+SP to treat uncomplicated malaria [17]. Altogether, studies conducted in Sudan between 2011 and 2015 detected high failure rates of treatment with AS-SP, ranging from $12.3 \%$ to $22.2 \%$. The evidence prompted a decision to change the new first-line of treatment to AL [18]. In 2017, Mohamed et al. reported a high rate of treatment failure against AS+SP which has led to the adoption of Dihydroartemisinin-piperaquine (DHA-PPQ) as the first-line treatment [19]. A year later, Hamid et al. (2018), stated that AS+SP is still effective against falciparum malaria in New halfa and Gezira Slanj regions [20].

$P$. falciparum chloroquine resistance transporter $(P f c r t)$ and $P$. falciparum multidrug resistance gene 1 (Pfmdr-1) are previously known membrane transporters associated with resistance to the drug combination of $C Q$ and $A Q$ or $M Q$ and Lumefantrine $(L)$ [21]. Also, with the suggestion of previous studies, point mutations in Pfmdr-1 are associated with the resistant to quinoline-containing drugs and artemisinin derivatives [21, 22]. Also, the presence of $P f m d r-1$ multicopy results in resistance to $M Q$ and $L$ $[23,24]$. However, the K76T mutation in Pfcrt mostly associated with the Pfcrt 72C, 73V, 74I, 75E, and 76T. The CVIET haplotype of $P f C r t$ is known as the most robust CQ resistance marker in Africa [12-15]. In vitro 
experiments showed that N86Y and Y184F mutations in the Pfmdr-1 gene increases the inhibitory concentrations of $C Q$ and $A Q[21,24]$, and reduce susceptibility to $M Q$ and L [24, 25].

Previous studies focused on the $P$. falciparum dihydrofolate reductase ( $P f d h f r)$ and dihydropteroate synthase (Pfdhps) which are known targets of pyrimethamine and sulfadoxine, respectively. Both drugs are inhibiting the folate pathway leading to the death of the parasite [19]. The emergence of resistant parasites has been documented in many parts of Africa [26]. Mutations in the Pfdhfr codons 51, 59, and 164 [16] and the substitutions at the Pfdhps in the codons 436, 437, 540, 581 and 613, suggested to be conferring resistance to sulfadoxine [19].

The need for updated molecular markers studies to investigate the frequency of falciparum malaria drugresistant is extreme. This study is aiming to describe the trend of $P$. falciparum drug resistance markers in 2017-2018 in comparison to CQ and AS/SP eras in Sudan.

\section{Methods}

A descriptive cross-sectional healthcare-centers based study conducted in Khartoum, Sennar, River Nile, Ad Damazin and Gedaref states between December 2017 and July 2018. Febrile patients (axillary temperature $<37 \mathrm{C}$ ) who were microscopically diagnosed with $P$. falciparum malaria infection were recruited to participate in the study after obtaining informed consent to collect the blood sample. Participants diagnosed with $P$. falciparum/ $P$. vivax co-infection and $P$. vivax mono-infections were excluded from the study. Two ml blood samples were collected prior to start treatment. No follow up data were obtained from patients after treatment. Blood samples were preserved into lithium heparin anticoagulation blood containers and shipped to the biotechnology lab, faculty of pharmacy at The Ahfad University for women for DNA extraction and molecular genotyping of drug resistance markers.

\section{DNA extraction}

The genomic DNA was extracted using the Guanidine Chloride extraction method described previously by Ciulla et al. (1988), with minor modifications [27]. In brief, $1 \mathrm{ml}$ from each blood sample was placed into $2.5 \mathrm{ml}$ tube and washed using RBCs washing buffer; and then nucleated and parasite cells were lysed using cell lysis buffer. $1 \mathrm{ml}$ of $6 \mathrm{M}$ Guanidine chloride solution and 10 micro of proteinase $\mathrm{K}$ enzyme were added and incubated over night at $37^{\circ} \mathrm{C}$. The following day, $2 \mathrm{ml}$ Chloroform were added to the mixture and centrifuged at $14000 \mathrm{rpm}$ for $10 \mathrm{~min} ; 3$ layers separation were formed. The upper layer containing the parasite and human DNA was transferred into new $2 \mathrm{ml}$ tubes; and $1 \mathrm{ml}$ absolute ethanol was added to precipitate the DNA. Precipitated DNA was then washed with $70 \%$ ethanol and allowed to dry for 1 hour before being dissolved with 200 micro distilled water. Purity and concentration of the extracted DNA was measured using Nanodrop 1000 spectrophotometer (USA). DNA was stored in $-20^{\circ} \mathrm{C}$ until molecular examinations later.

\section{Parasite genotyping and assessment of multiple infection}


To confirm the microscopic diagnosis, the protocol and primers sets described previously by Snounou et al. (1993) were used [28]. Whereas, for assessment of multiple infection, primers and PCR conditions used were according to the previously described protocol [29].

\section{Drug resistance markers assessment}

Genotyping of the specific point mutations in the $P$. falciparum genome was done using Sanger sequencing method using the primers sets for the Pfcrt, Pfmdr-1, Pfdhfr, and Pfdhps genes as described previously [30]. Primers sets used in this study were described in table 1. PCR amplification conditions were set to $95^{\circ} \mathrm{C}$ for $5 \mathrm{~min}$ as initial denaturation followed by 35 cycles of $95^{\circ} \mathrm{C}$ for $1 \mathrm{~min}, 55^{\circ} \mathrm{C}$ for $1 \mathrm{~min}$ (for Pfcrt, Pfmdr-1) or $58^{\circ} \mathrm{C}$ for $1 \mathrm{~min}$ (for Pfdhfr and Pfdhps), and $72^{\circ} \mathrm{C}$ for $1 \mathrm{~min}$, followed by final extension step at $72^{\circ} \mathrm{C}$ for $10 \mathrm{~min}$. All thermal conditions were performed in $\mathrm{MJ}$ thermocycler PCR machine (USA). PCR amplicons were then sequenced in both directions using the forward and reverse primer for each gene to exclude any base calling errors that could be obtained during sequencing. Sequences were validated using GENtle software (v1.9.4) and aligned in comparison with the wildtype $P$. falciparum 3D7 strain reference sequences (PF3D7_0709000 for Pfcrt, PF3D7_0523000 for Pfmdr-1, PF3D7_1324800 for Pfdhfr, and PF3D7_0810800 for Pfdhps). The deduced amino acids were translated from nucleotide sequences using MEGA7 software (v7.0.26) using the standard translation code in order to determine sequences mutations at the Pfcrt codons; 72, 73, 74, 75, and 76; Pfmdr-1 codons 86 and 184; Pfdhfr codons 51, 59, and 164; and the substitutions at the Pfdhps in the codons 436, 437, 540, 581 and 613.

\section{Previous reports on drug resistance markers}

Data deposited by the worldwide antimalarial resistance network (WWARN) (https://www.wwarn.org/) was consulted and the molecular markers previously reported from Sudan were identified, collected, and analyzed to compare between past and present frequency of malaria drug resistance mutations. Data sets included SP molecular surveyors (https://www.wwarn.org/sp-molecular-surveyor), and ACT partner drug molecular surveyors (https://www.wwarn.org/tracking-resistance/act-partner-drug-molecularsurveyor). Numbers and drug molecular marker genotypes of $P$. falciparum isolates included in the historical literature review data set analyzed in this study are presented in Supplementary file 1.

\section{Statistical analysis}

The statistical analysis was done using the statistical Package for Social Sciences (SPSS, v20.0). Oneway ANOVA test was used to calculate the least significance difference (LSD) of frequency distribution in the molecular markers collected from the previous reports from Sudan in comparison to this study. Pearson correlation was used to investigate the association between the different drug resistance markers. $P$ value $\leq 0.05$ was considered significant.

\section{Results}




\section{The molecular genotyping of the field-isolated $P$. falciparum and multiclonal infections assessment}

In this small-scale study, a total of 28 malaria parasite isolates were genotyped, of them, 2 and 6 isolates were excluded since were $P$. falciparum/ $P$. vivax coinfections, $P$. vivax infection, respectively. The remaining 20 isolates were $P$. falciparum mono-infections. Multiclonal infections assessment for the 20 isolates were $16(80 \%)$ MAD20, $2(10 \%) \mathrm{K} 1$, and $2(10 \%) \mathrm{RO} 33$, based on the merozoite surface protein 1 (MSP-1) gene.

\section{Frequency of $P$. falciparum drug resistance markers}

Drug molecular markers analysis was successfully done on the $20 P$. falciparum isolates. Out of the 20 isolates Pfcrt K76 showed the highest frequency; 16 (80\%). Pfcrt 76T was 4 (20\%). None of the isolates were carrying mixed Pfcrt allele infection. For the Pfmdr-1 marker, $9(45 \%)$ isolates were carrying the N86 allele and 11 (55\%) were carrying the $86 \mathrm{Y}$ allele. While the Y184F of the Pfmdr-1 showed higher frequency of $184 \mathrm{~F}$ compared to Y184; 16 (80\%) and 4 (20\%), respectively. Concerning the double Pfmdr-1 haplotype, NY haplotype was $2(10 \%)$, NF was $7(35 \%)$, YF was $9(45 \%)$, and YY was $2(10 \%)$.

The Pfdhfr N51I showed higher frequency of 51 I compared to N51; 18 (90\%) and 2 (10\%), respectively. Whereas for C59R, C59 was 18 (90\%), and 59R was 2 (10\%). For S108N, 18 (90\%) for 108N and 2 (10\%) for S108. For the triplet haplotype of the Pfdhfr, the haplotype ICN was the most frequent haplotype; 16 (80\%). IRN and NCS were only present in two isolates; 2 (10\%) for each.

Concerning the Pfdhps drug molecular marker, all the 20 (100\%) isolates were carrying the mutant alleles; 437G and 540E. the Pfdhps haplotype present was the double GE haplotype only (Table 2).

No statistically significant correlation was found for the Pfcrt, Pfmdr-1, Pfdhfr, and Pfdhps. However, a statistically significantly positive correlation was observed for Pfmdr-1 and the combined Pfdhfr and Pfdhps alleles, Pearson $r^{\prime}=0.509, \mathrm{P}$ value $=0.035$. While, for the Pfcrt and the combined Pfdhfr and Pfdhps, statistically insignificant negative correlation was found, Pearson $r^{\prime}=-0.248, P$ value $=0.291$. Pfdhps was not correlated separately with any of the other mutations since present in all the studied samples.

\section{The trend in $P$. falciparum multidrug resistance from 1989 to 2018 according to location}

Reports on P. falciparum multidrug resistant collected from 1989 to 2016 showed extreme fluctuation. High prevalence of Pfcrt 76T allele was observed in Khartoum throughout all years of previous studies. However, in the current study the PfcrtK76 is very high compared to the previous years. While in Gedaref Pfcrt 76T showing increased prevalence each year, reaching $89 \%$ in the study conducted in 2007 . The Pfcrt 76T allele during the previous studies is showing higher prevalence compared to Pfcrt K76. The situation of the increase in $P$. falciparum resistance markers among the previous studies was relatively similar. All studied genes showing increase prevalence of the mutant alleles and a reduction of the wildtype alleles. However, in Khartoum the Pfdhfr IN and Pfdhps GE haplotypes prevalence in 2016 and 2018 isolates constitute nearly all the studied samples comparing to other locations (Fig. 1). 


\section{The trend in $P$. falciparum multidrug resistance from 1989 to 2018 in Sudan}

For the $P f c r t$, the T allele that confers CQ resistant was at higher frequency during 2000-2001 (89.6\%), however, T mutant allele frequency started to dropdown reaching up to $43.9 \%$ in 2016 and bottomed at $20 \%$ in 2018 . In this study, the frequency of the K76 wildtype allele was higher compared to all previous years; $80 \%$. Whereas, the N86Y mutation of the Pfmdr-1 was extremely flocculation during the past years (Fig. 2).

Concerning the Pfdhfr N51I and S108N, in 1996-1997 the NS wildtype haplotype was showing low frequency compared to the IN mutant haplotype; $18.6 \%$ and $74.3 \%$. while in 1998-1999, frequency of the NS haplotype reached to $100 \%$. The prevalence of the NS haplotype from 2002-2003 continued to decrease down to $10 \%$ in $2017-2018$. On the other hand, the IN mutant haplotype increased to $85.5 \%$ in 2002-2003 reaching $92.7 \%$ in 2009-2012 and remained constant approximately at $90 \%$ in 2017-2018. However, for the Pfdhps, in 1998-1999 AK wildtype haplotype was 93.1\%, but in 2002-2003 GE mutant haplotype increased to $75.1 \%$. in 2007 the AK wildtype haplotype increased again to $77.8 \%$ and decreased to $36.1 \%$ in $2009-2012$ and $51.1 \%$ in 2016 . However, in this study in 2017-2018, the GE mutant haplotype was prevalent in all the studied samples 20 (100\%) (Fig. 3).

The prevalence of the Pfmdr-1 double haplotype N86Y and Y184F was only obtained from 4 previous studies. Results of Pfmdr-1 double haplotype in comparison with this study showed an increase in the NY mutant haplotype in $2017-2018$ compared to $2016 ; 45 \%$ and $31.5 \%$, respectively. While noted a quite reduction of the NY and YY wildtype haplotypes throughout all years (Fig. 4).

Frequency distribution of the Pfcrt K76T and Pfmdr-1 N86Y alleles, Pfmdr-1; N86Y and Y184F, Pfdhfr, N51I and S108N, and Pfdhps; A437G and K540E double haplotypes was significantly different across the whole years in Sudan. an illustrated statistical significance and insignificance of frequency distribution of $P$. falciparum multidrug resistance markers between the different years intervals is described in supplementary file 2; (tables S1-S5).

\section{Discussion}

Studying the molecular markers to monitor the prevalence of drug resistance is extremely beneficial especially for public health programs that aim to reduce the prevalence of malaria infections because drug-resistant parasites are the major threat to achieve success malaria control/elimination. In this study we aimed to investigate the fluctuation in the $P$. falciparum multidrug resistance markers in Sudan during $\mathrm{CQ}, \mathrm{AS} / \mathrm{SP}$ and ACT eras. In the current small-scale study, the reported frequency of Pfcrt K76 wildtype allele in the filed-isolates was very high compared to all previous years, especially during the years of CQ deployment in Sudan [16]. However, since AS/SP era, post-CQ in 2004, Pfcrt K76 allele tends to increase. This could be occurred due to limited exposure of the $P$. falciparum parasite to the CQ or the complete absence of the pressure since infections with $P$. vivax or $P$. falciparum / $P$. vivax coinfection is been treated with combination of AL and PQ [1]. However, this increase in Pfcrt K76 might be due to the increase of AL 
pressure. Similar results supporting the reasons for the increase of Pfcrt K76 allele were also reported from China [31].

In 2003, molecular markers studies confirmed that CQ was no longer suitable for malaria treatment and SP treatment failure was only ranging between $1.9 \%$ and $7.8 \%$, this led to the change of treatment guidelines in 2004 [7, 32-34]. At the same time, the increase in Pfdhfrand Pfdhps double mutant haplotypes was noted in the following years [16]. The Pfdhfr IN haplotype and the Pfdhps GE haplotype which both confers the resistant to SP, increased significantly by 2007 [35], In 2017-2018, both haplotypes were showing higher frequency; $90.0 \%$ and $100 \%$. This could be due to the continued encountered pressure at the Pfdhfr and Pfdhps genes in the parasite, in parallel to the reduced Pfcrt 76T allele since CQ-pressure is limited. This phenomenon is observed significantly in all countries in which AS/SP was used as first-line therapy for malaria infections [36].

The prevalence of the Pfmdr-1 NF haplotype in 2017-2018 which could confer the resistant to AL is noted among $35 \%$ of the study samples. Comparison between previous years for the frequency distribution of Pfmdr-1 double haplotypes showed a statistically significant difference between all years and 2008; P values 0.000 for 2009 to 2012 and 2016, and 0.005 for 2017 to 2018, when at that time the recommended malaria treatment was AS/SP, and since 2009 the use of AL is significantly increased due to malpractice in drug use, such as usage of incorrect dosage and insufficient information stated to patients about the prescribed treatment which may lead to the increase in resistance and recurrent infections rates [37].

Although, in this study the quintuple NFSND haplotype of the Pfmdr-1 is not completely studied, but this is also considered alarming since the presence of NF haplotype could be accompanied with the SND haplotype, and consequently leading to AL resistance since parasites carrying the NFSND haplotype reported to survive 15-fold higher AL concentrations [22]. Previously, development of molecular markers for AL resistance was thought to be difficult because there were no known resistant lab lines can be used as resistance controls, but with the ability to predict with the aid of the Genome-wide Analytical Study (GWAS) [38], this gave the chance to investigate further genes that could develop mutations under treatments pressure such as kelch13 and plasmepsins 2-3 multicopy at the same time investigating the Pfmdr-1 NFSND haplotype could be the role evolution for the developed mutations since allow longer survival rate of the parasite $[22,39]$.

Although, as previously described by Wang et al., 1997, established the association of Pfdhfrand Pfdhps haplotypes with SP resistance [40]. In the present study, Pfmdr-1 86Y allele, Pfdhfr IN haplotype and Pfdhps GE haplotype were consisting the majority of the studied samples; $90 \%$. This however, is also similar to previous study conducted in Sudan where all the parasite isolates carrying the Pfcrt 76T and Pfmdr-1 86Y mutant alleles were carrying the mutant Pfdhfr IN haplotype and Pfdhps 540E mutation [35]. But difference obtained in this study is that most of the isolates were carrying Pfcrt K76 wildtype allele. As this is in accordance to stop the use of CQ in malaria treatment. As well, the prevalence of Pfdhps double haplotype GE could hinder the effect of SP if used as Intermittent preventive therapy during 
pregnancy (IPTp). Although, SP as IPTp was not implemented and there is scarce information about the use of SP during pregnancy in Sudan (Mohamed et al., unpublished data). And the presence of Pfdhfr IRN in combination with Pfdhps GE haplotypes forming the quintuple mutant haplotype confer a high risk for treatment failure in malaria-infected children and nonpregnant adults who receive SP as a seasonal malaria chemo-prevention treatment (SMC-SP) [41]. However, previous studies indicated that IPTp-SP is still efficacious in some areas with high prevalence of resistant $P$. falciparum parasite [42]. Nevertheless, the increased resistance rate might compromise the implication of IPTp-SP [43-45].

In this study, a small frequency of R59 mutation was observed, this could be attributed to the small sample size investigated particularly that the R59 mutation is quite known throughout most of the African countries. However, comparing to previously reported studies in Sudan, this frequency is similar, as suggested previously, this could be due to high frequency of Pfdhfr $51 \mathrm{I}$ and $108 \mathrm{~N}$, and Pfdhps $437 \mathrm{G}$ and 540E mutations lead to a genetical cost-effectiveness competitions between the multi-drugsresistance mutations within the parasites for determination of the effective combination of mutations that increase the parasite survival rate [46].

\section{Conclusions And Recommendations}

In addition to studying the molecular markers of malaria multi-drug resistance in the field collected isolates of $P$. falciparum we reviewed and summarized the previously published reports about malaria drugs resistance in Sudan. This study describes the distribution of $P$. falciparum multidrug resistance markers throughout Sudan. It also provides a solid baseline data of the status of these markers which could be very useful for the malaria control program not only for establishing surveillance system that monitor the change in and/or the emergence of malaria drug resistance but it will also offer a guidance for the evidence-base decision-making regarding the treatment protocol national and regional wise. Nevertheless, a country-wide monitoring and evaluation program for the early detection of the drugresistance is extremely needed for more effective treatment protocol and a successful control of the disease.

\section{List Of Abbreviations}

ACTs; Artemisinin-based Combination Treatment

AL; Artemether-Lumefantrine

AQ; Amodiaquine-Hydrochloride

AS; Artesunate

CQ; Chloroquine

DHA-PPQ; Dihydroartemisinin-Piperaquine 
GWAS; Genome-Wide Analytical Study

IPTp; Intermittent Preventive Therapy during Pregnancy

L; Lumefantrine

MSP-1; Merozoite Surface Protein 1

MQ; Mefloquine

P; Pyrimethamine

Pfcrt, P. falciparum Chloroquine Resistance Transporter

Pfdhfr, P. falciparum Dihydrofolate Reductase

Pfdhps, P. falciparum Dihydropteroate Synthase

Pfmdr-1; P. Falciparum Multidrug Resistance Gene 1

SMC-SP; Seasonal Malaria Chemo-Prevention Treatment

SP; Sulfadoxine-Pyrimethamine

TES; Therapeutic Efficacy Studies

WWARN; Worldwide Antimalarial Resistance Network

\section{Declarations}

Ethics approval and consent to participate

This study was reviewed and approved by the National University Biomedical Research Ethics Committee, National University-Sudan. Oral and written informed consents were obtained from participants' or parents or guardians in case of children.

Consent for publication

Not applicable

Availability of data and material

All datasets used and analysed in this study are available in the manuscript. Sequences analysed in this study were not submitted into the NCBI database.

Competing interests 
The authors declare that they have no competing interests

Funding

Not applicable

Author's contributions

NSM and CHS conceived, designed the study protocol, HA, HAO, AEA, AMY, YBE, EYO, ARE, MMO and EES carried out the parasitological and molecular examinations. MM, AAM, YA, AA, MMO, MSA, and RAO conducted the genetic and molecular markers analysis. NSM, HA, HAO, AEA and EES analysed and interpreted the data. NSM, AA, MSA, and RAO designed and supervised the experiments. NSM, AA, and CHS drafted the manuscript. All authors read and approved the final manuscript.

Acknowledgements

We are of great thanks for kind collaboration and assistance of the clinical staff of all hospitals and health centers for providing the samples. Also, great thanks to all participants contributed to this work.

\section{References}

1. WHO: World malaria report 2019. Geneva: World Health Organization 2019.

2. Abbott P: Trials with Gamoquin and Daraprim in the Treatment of Malaria at Wad Medani, Sudan. Sudan Medical Journal 1955, 1:33-38.

3. Kouznetsov R, Rooney W, Wernsdorfer W, El Gaddal A, Payne D, Abdalla R: Assessment of the sensitivity of Plasmodium falciparum to antimalarial drugs at Sennar, Sudan: use of the in vitro micro-technique and the in vivo method. Geneva: World Health Organization; 1979.

4. Babiker HA, Creasey AM, Fenton B, Bayoumi RA, Arnot DE, Walliker D: Genetic diversity of Plasmodium falciparum in a village in eastern Sudan. 1. Diversity of enzymes, 2D-PAGE proteins and antigens. Transactions of the Royal Society of Tropical Medicine Hygiene 1991, 85:572-577.

5. Ibrahim ME, Awad-El-Kariem FM, El Hassan IM, El Rashid M: A case of Plasmodium falciparum malaria sensitive to chloroquine but resistant to pyrimethamine/sulfadoxine in Sennar, Sudan. Transactions of the Royal Society of Tropical Medicine Hygiene 1991, 85:446.

6. Awad-El-Kariem F, Miles M, Warhurst D: Chloroquine-resistant Plasmodium falciparum isolates from the Sudan lack two mutations in the pfmdr1 gene thought to be associated with chloroquine resistance. Transactions of the Royal Society of Tropical Medicine Hygiene 1992, 86:587-589.

7. Khalil I, Rønn AM, Alifrangis M, Gabar HA, Satti GM, Bygbjerg IC: Dihydrofolate reductase and dihydropteroate synthase genotypes associated with in vitro resistance of Plasmodium falciparum to pyrimethamine, trimethoprim, sulfadoxine, and sulfamethoxazole. The American journal of tropical medicine hygiene 2003, 68:586-589. 
8. Ochong EO, van den Broek IV, Keus K, Nzila A: association between chloroquine and amodiaquine resistance and allelic variation in the Plasmodium falciparum multiple drug resistance 1 gene and the chloroquine resistance transporter gene in isolates from the upper Nile in southern Sudan. The American journal of tropical medicine hygiene 2003, 69:184-187.

9. Abdel-Muhsin A, Mackinnon M, Awadalla P, Ali E, Suleiman S, Ahmed S, Walliker D, Babiker HA: Local differentiation in Plasmodium falciparum drug resistance genes in Sudan. Parasitology 2003, 126:391-400.

10. Alifrangis M, Enosse S, Khalil IF, Tarimo DS, Lemnge MM, Thompson R, Bygbjerg IC, Rønn AM: Prediction of Plasmodium falciparum resistance to sulfadoxine/pyrimethamine in vivo by mutations in the dihydrofolate reductase and dihydropteroate synthetase genes: a comparative study between sites of differing endemicity. The American journal of tropical medicine hygiene 2003, 69:601-606.

11. Malik EM, Khalafalla O: Malaria in Sudan: past, present and the future. Gezira journal of health sciences 2004, 1.

12. Adam I, Ibrahim M, Aelbasit I, El Bashir M: Efficacy of sulfadoxine/pyrimethamine for uncomplicated Plasmodium falciparum malaria in a small sample of Sudanese children. 2004.

13. Salah MT, Mohammed MM, Himeidan YE, Malik EM, Elbashir MI, Adam I: A randomized comparison of sulphadoxine-pyrimethamine and combination of sulphadoxine pyrimethamine with chloroquine in the treatment of uncomplicated falciparum malaria in Eastern Sudan. Saudi medical journal 2005, 26:147-148.

14. Elamin SB, Malik EM, Abdelgadir T, Khamiss AH, Mohammed MM, Ahmed ES, Adam I: Artesunate plus sulfadoxine-pyrimethamine for treatment of uncomplicated Plasmodium falciparum malaria in Sudan. Malaria Journal 2005, 4:41.

15. Adam I, A-Elbasit I, Idris S, Malik E, Elbashir M: A comparison of the efficacy of artesunate plus sulfadoxine-pyrimethamine with that of sulfadoxine-pyrimethamine alone, in the treatment of uncomplicated, Plasmodium falciparum malaria in eastern Sudan. Annals of Tropical Medicine Parasitology 2005, 99:449-455.

16. Adeel AA: Drug-resistant malaria in Sudan: a review of evidence and scenarios for the future. Sudanese journal of paediatrics 2012, 12:8.

17. Adeel AA, Elnour FAA, Elmardi KA, Abd-Elmajid MB, Elhelo MM, Ali MS, Adam MA, Atta H, Zamani G, Warsame M: High efficacy of artemether-lumefantrine and declining efficacy of artesunate+ sulfadoxine-pyrimethamine against Plasmodium falciparum in Sudan (2010-2015): evidence from in vivo and molecular marker studies. Malaria journal 2016, 15:285.

18. Organization WH: World Malaria Day 2018" Ready to beat malaria": key messages. World Health Organization; 2018.

19. Mohamed AO, Hamid MMA, Mohamed OS, Elkando NS, Suliman A, Adam MA, Elnour FAA, Malik EM: Efficacies of DHA-PPQ and AS/SP in patients with uncomplicated Plasmodium falciparum malaria in an area of an unstable seasonal transmission in Sudan. Malar J 2017, 16:163. 
20. Hamid MMA, Thriemer K, Elobied ME, Mahgoub NS, Boshara SA, Elsafi HM, Gumaa SA, Hamid T, Abdelbagi $\mathrm{H}$, Basheir HM: Low risk of recurrence following artesunate-Sulphadoxinepyrimethamine plus primaquine for uncomplicated Plasmodium falciparum and Plasmodium vivax infections in the Republic of the Sudan. Malaria journal 2018, 17:117.

21. Duraisingh MT, Roper C, Walliker D, Warhurst DC: Increased sensitivity to the antimalarials mefloquine and artemisinin is conferred by mutations in the pfmdr1 gene of Plasmodium falciparum. Molecular microbiology 2000, 36:955-961.

22. Malmberg M, Ngasala B, Ferreira PE, Larsson E, Jovel I, Hjalmarsson A, Petzold M, Premji Z, Gil JP, Bjorkman A, Martensson A: Temporal trends of molecular markers associated with artemetherlumefantrine tolerance/resistance in Bagamoyo district, Tanzania. Malar J 2013, 12:103.

23. Ecker A, Lehane AM, Fidock DA: Molecular markers of Plasmodium resistance to antimalarials. In Treatment and Prevention of Malaria. Springer; 2011: 249-280

24. Price RN, Uhlemann A-C, Brockman A, McGready R, Ashley E, Phaipun L, Patel R, Laing K, Looareesuwan S, White NJ: Mefloquine resistance in Plasmodium falciparum and increased pfmdr1 gene copy number. The Lancet 2004, 364:438-447.

25. Mukherjee A, Gagnon D, Wirth DF, Richard D: Inactivation of plasmepsins 2 and 3 sensitizes Plasmodium falciparum to the antimalarial drug piperaquine. Antimicrobial agents chemotherapy 2018, 62:e02309-02317.

26. Takata J, Sondo P, Burrow R, Maguire B, Hossain S, Das D, Commons R, Price R, Guerin P: The wwarn clinical trials publication library: a systematically constructed database of plasmodium clinical efficacy trials. 2019.

27. Ciulla TA, Sklar RM, Hauser SL: A simple method for DNA purification from peripheral blood. Analytical biochemistry 1988, 174:485-488.

28. Snounou G, Viriyakosol S, Jarra W, Thaithong S, Brown KN: Identification of the four human malaria parasite species in field samples by the polymerase chain reaction and detection of a high prevalence of mixed infections. Molecular biochemical parasitology 1993, 58:283-292.

29. Ntoumi F, Ngoundou-Landji J, Lekoulou F, Luty A, Deloron P, Ringwald P: Site-based study on polymorphism of Plasmodium falciparum MSP-1 and MSP-2 genes in isolates from two villages in Central Africa. Parassitologia 2000, 42:197-203.

30. Salem OA, Mint Lekweiry K, Bouchiba H, Pascual A, Pradines B: Ould Mohamed Salem Boukhary A, et al. Characterization of Plasmodium falciparum genes associated with drug resistance in Hodh Elgharbi, a malaria hotspot near Malian-Mauritanian border. Malar J 2017, 16:140.

31. Lu F, Zhang M, Culleton RL, Xu S, Tang J, Zhou H, Zhu G, Gu Y, Zhang C, Liu Y, et al: Return of chloroquine sensitivity to Africa? Surveillance of African Plasmodium falciparum chloroquine resistance through malaria imported to China. Parasit Vectors 2017, 10:355.

32. Abdel Hameed A: Antimalarial drug resistance in the Eastern Mediterranean Region. 2003.

33. Abdel-Hameed A, El-Jak I, Faragalla I: Sentinel posts for monitoring therapeutice efficacy of antimalarial drugs against Plasmodium falciparum infections in the Sudan. African journal of 
medicine medical sciences 2001, 30:1-5.

34. van den Broek IV, Gatkoi T, Lowoko B, Nzila A, Ochong E, Keus K: Chloroquine, sulfadoxinepyrimethamine and amodiaquine efficacy for the treatment of uncomplicated Plasmodium falciparum malaria in Upper Nile, south Sudan. Transactions of the Royal Society of Tropical Medicine Hygiene 2003, 97:229-235.

35. Osman ME, Mockenhaupt FP, Bienzle U, Elbashir MI, Giha HA: Field-based evidence for linkage of mutations associated with chloroquine (pfcrt/pfmdr1) and sulfadoxine-pyrimethamine (pfdhfr/pfdhps) resistance and for the fitness cost of multiple mutations in P. falciparum. Infect Genet Evol 2007, 7:52-59.

36. Flegg JA, Metcalf CJE, Gharbi M, Venkatesan M, Shewchuk T, Hopkins Sibley C, Guerin PJ: Trends in antimalarial drug use in Africa. Am J Trop Med Hyg 2013, 89:857-865.

37. Elmannan AA, Elmardi KA, Idris YA, Spector JM, Ali NA, Malik EM: Anti-malarial prescribing practices in Sudan eight years after introduction of artemisinin-based combination therapies and implications for development of drug resistance. BMC Pharmacol Toxico/ 2015, 16:3.

38. Amato R, Lim P, Miotto O, Amaratunga C, Dek D, Pearson RD, Almagro-Garcia J, Neal AT, Sreng S, Suon S, et al: Genetic markers associated with dihydroartemisinin-piperaquine failure in Plasmodium falciparum malaria in Cambodia: a genotype-phenotype association study. Lancet Infect Dis 2017, 17:164-173.

39. Bakhiet AMA, Abdelraheem MH, Kheir A, Omer S, Gismelseed L, Abdel-Muhsin AA, Naiem A, Al Hosni A, Al Dhuhli A, Al Rubkhi $M$, et al: Evolution of Plasmodium falciparum drug resistance genes following artemisinin combination therapy in Sudan. Trans R Soc Trop Med Hyg 2019, 113:693-700.

40. Wang P, Lee CS, Bayoumi R, Djimde A, Doumbo O, Swedberg G, Dao LD, Mshinda H, Tanner M, Watkins WM, et al: Resistance to antifolates in Plasmodium falciparum monitored by sequence analysis of dihydropteroate synthetase and dihydrofolate reductase alleles in a large number of field samples of diverse origins. Mol Biochem Parasito/ 1997, 89:161-177.

41. Kublin JG, Dzinjalamala FK, Kamwendo DD, Malkin EM, Cortese JF, Martino LM, Mukadam RA, Rogerson SJ, Lescano AG, Molyneux ME: Molecular markers for failure of sulfadoxinepyrimethamine and chlorproguanil-dapsone treatment of Plasmodium falciparum malaria. The Journal of infectious diseases 2002, 185:380-388.

42. Organization WH: Evidence Review Group: Intermittent Preventive Treatment of Malaria in Pregnancy (IPTp) with Sulfadoxine-Pyrimethamine (SP). Geneva: WHO. 2012.

43. Harrington WE, Morrison R, Fried M, Duffy PE: Intermittent preventive treatment in pregnant women is associated with increased risk of severe malaria in their offspring. PLoS One 2013, 8:e56183.

44. Shulman C, Dorman E, Cutts F, Kawuondo K, Bulmer J, Peshu N, Marsh K: Intermittent sulphadoxinepyrimethamine to prevent severe anaemia secondary to malaria in pregnancy: a randomised placebo-controlled trial. The Lancet 1999, 353:632-636.

45. Harrington W, Mutabingwa T, Muehlenbachs A, Sorensen B, Bolla M, Fried M, Duffy P: Competitive facilitation of drug-resistant Plasmodium falciparum malaria parasites in pregnant women who 
receive preventive treatment. Proceedings of the National Academy of Sciences 2009, 106:9027-

9032.

46. Group WKG-PS: Association of mutations in the Plasmodium falciparum Kelch13 gene (Pf3D7_1343700) with parasite clearance rates after artemisinin-based treatments-a WWARN individual patient data meta-analysis. BMC Med 2019, 17:1.

\section{Tables}

Table 1: Primers sets used for the amplification of the drug-target genes

\begin{tabular}{|c|c|c|}
\hline Primer name & Primer sequence & $\begin{array}{l}\text { Annealing } \\
\text { temperature }\end{array}$ \\
\hline $\begin{array}{l}\text { Pfcrt } 1 \text { forward } \\
\text { Pfcrt } 1 \text { reverse }\end{array}$ & $\begin{array}{l}5^{\prime} \text {-GTT CTT GTC TTG GTA AAT GT-3' } \\
5^{\prime} \text { - CGG ATG TTA CAA AAC TAT AGT T-3' }\end{array}$ & $55^{\circ} \mathrm{C}$ \\
\hline $\begin{array}{l}\text { Pfmdr-1 } \\
\text { forward }\end{array}$ & 5'-AGA GAA AAA AGA TGG TAA CCT CAG-3' & $55^{\circ} \mathrm{C}$ \\
\hline $\begin{array}{l}\text { Pfmdr-1 } \\
\text { reverse }\end{array}$ & 5'-ACC ACA AAC ATA AAT TAA CGG-3' & \\
\hline Pfdhfr forward & 5'-TTC TCC TTT TTA TGA TGG AAC AAG T-3' & $58^{\circ} \mathrm{C}$ \\
\hline Pfdhfr reverse & 5' ATA TTT GAA AAT CAT TTG GAT GTA TAG-3' & \\
\hline Pfdhps forward & $\begin{array}{l}\text { 5'-TGC TTA AAT GAT ATG ATA CCC GAA TAT AAG- } \\
3^{\prime}\end{array}$ & $58^{\circ} \mathrm{C}$ \\
\hline Pfdhps reverse & $5^{\prime}$ TCC ACC TGA AAA GAA ATA CAT AAA T-3' & \\
\hline
\end{tabular}

Table 2: The distribution of multidrug resistance markers among the 2017-2018 study isolates.

\begin{tabular}{lccccc}
\hline Isolate ID & Pfcrt & Pfmdr-1 & Pfdhfr & Pfdhps & Combined* \\
\hline Isolate 1 & K & YF & ICN & GE & KYFICNGE \\
Isolate 2 & T & NF & ICN & GE & TNFICNGE \\
\hline Isolate 3 & K & YF & ICN & GE & KYFICNGE \\
\hline Isolate 4 & K & YY & ICN & GE & KYYICNGE \\
\hline Isolate 5 & T & NF & ICN & GE & TNFICNGE \\
\hline Isolate 6 & K & NY & ICN & GE & KNYICNGE \\
\hline Isolate 7 & K & YF & IRN & GE & KYFIRNGE \\
\hline Isolate 8 & K & YF & NCS & GE & KYFNCSGE \\
\hline Isolate 9 & K & NY & ICN & GE & KNYICNGE \\
\hline Isolate 10 & T & NF & ICN & GE & TNFICNGE \\
\hline Isolate 11 & K & NF & ICN & GE & KNFICNGE \\
\hline Isolate 12 & K & YF & NCS & GE & KYFNCSGE \\
\hline Isolate 13 & K & NF & ICN & GE & KNFICNGE \\
\hline Isolate 14 & K & YF & IRN & GE & KYFIRNGE \\
\hline Isolate 15 & T & NF & ICN & GE & TNFICNGE \\
\hline Isolate 16 & K & YF & ICN & GE & KYFICNGE \\
\hline Isolate 17 & K & YF & ICN & GE & KYFICNGE \\
\hline Isolate 18 & K & YY & ICN & GE & KYYICNGE \\
\hline Isolate 19 & K & YF & ICN & GE & KYFICNGE \\
\hline Isolate 20 & K & NF & ICN & GE & KNFICNGE \\
\hline
\end{tabular}


*Letters denotes the wildtype and mutant alleles of the Pfcrt K76T; Pfmdr-1 N86Y and Y184F; Pfdhfr N51I, C59R, and S108N; Pfdhps A437G and K540E.

Figures
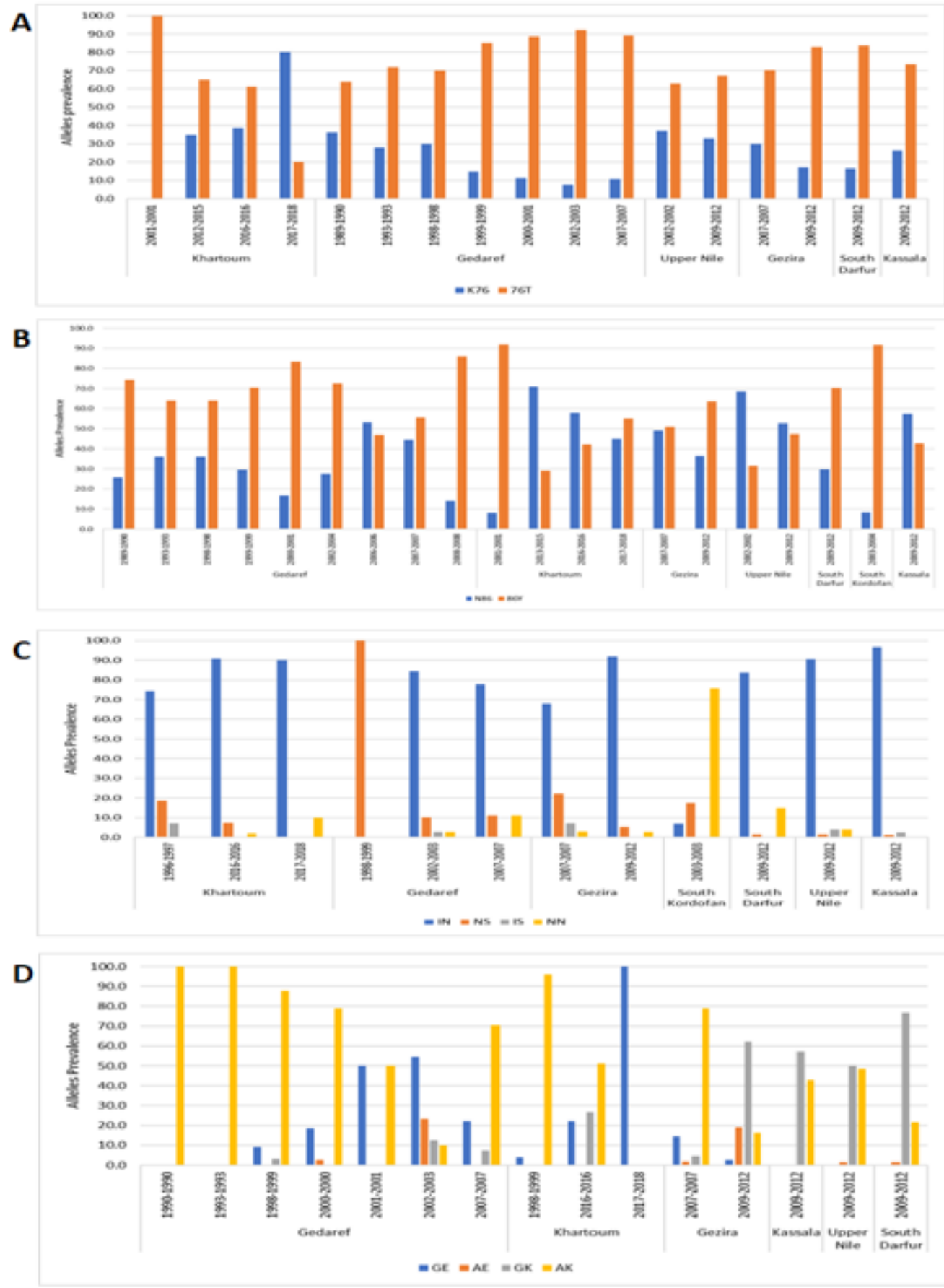

\section{Figure 1}

Prevalence of P. falciparum drug resistance markers from 1989 to 2018 according to location. A: Prevalence of Pfcrt K76T alleles. B: Prevalence of Pfmdr-1 N86Y alleles. C: Prevalence of Pfdhfr double haplotypes N51I and S108N. D: Prevalence of Pfdhps double haplotypes A437G and K540E. 


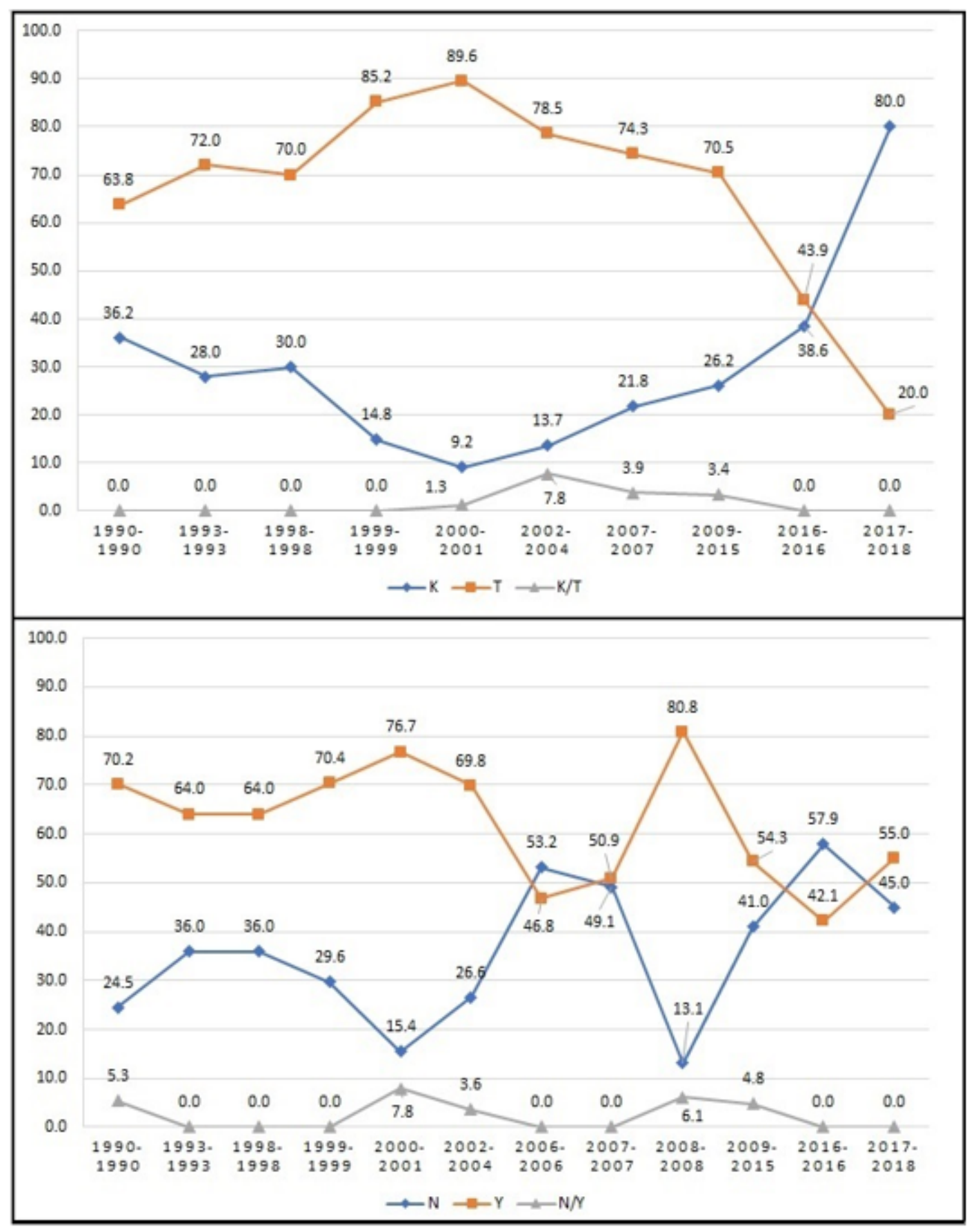

Figure 2

Frequency distribution of single Pfcrt K76T and Pfmdr-1 N86Y genotypes in 2017-2018 samples compared with previously published reports. 


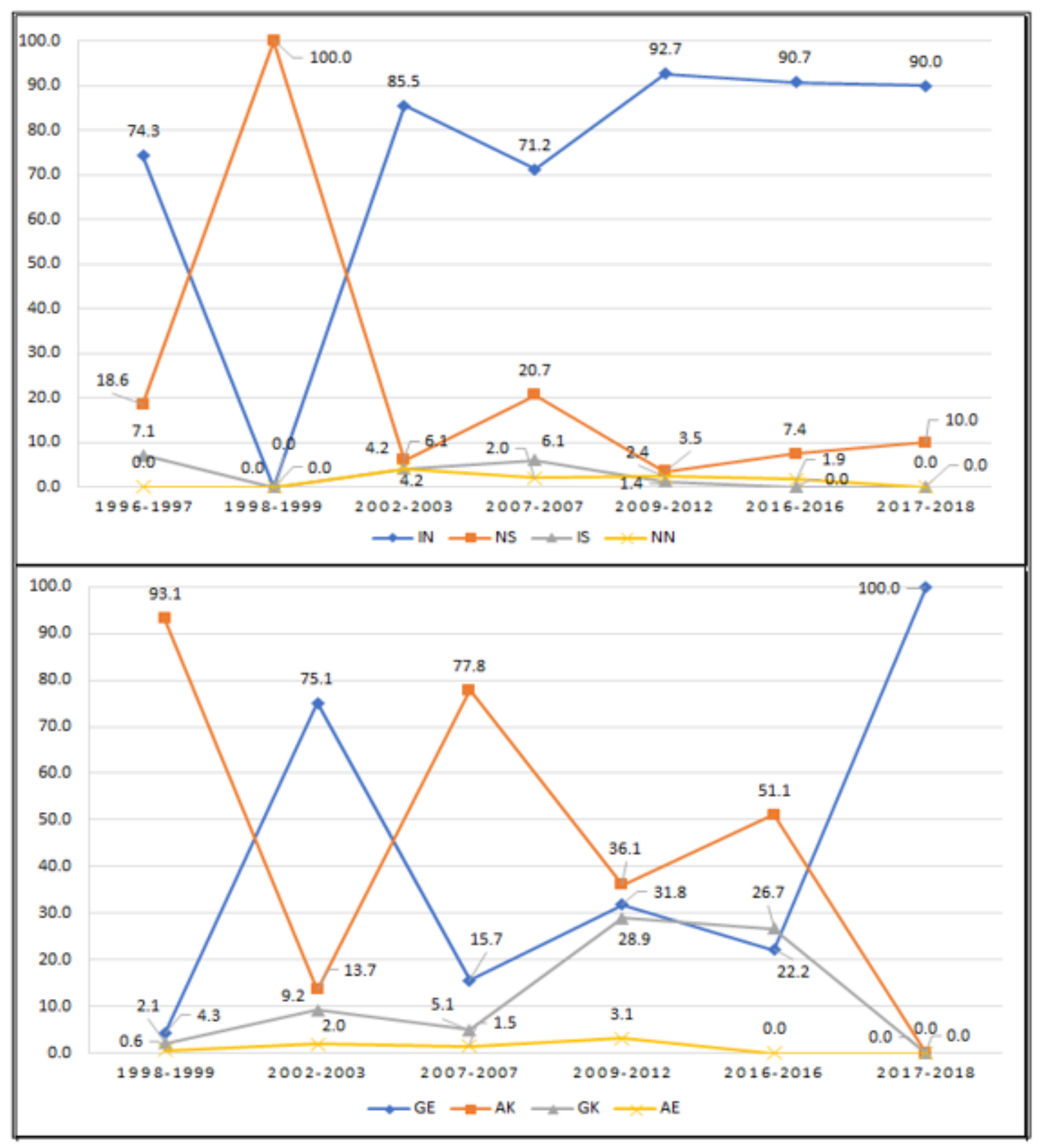

Figure 3

Frequency distribution of double haplotypes of Pfdhfr N51I and S108N, and Pfdhps A437G and K540E in 2017-2018 samples compared with previously published reports. 


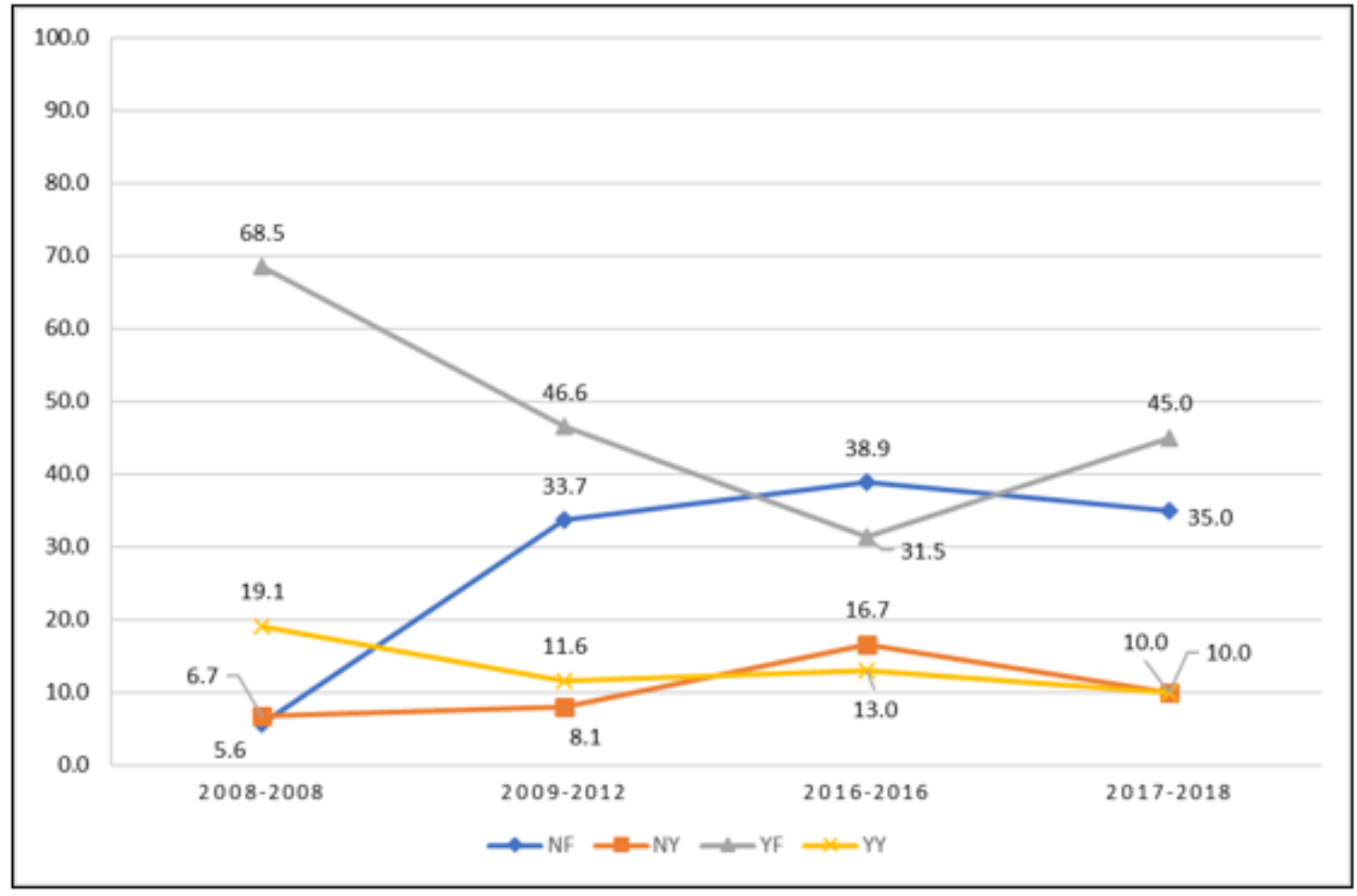

Figure 4

Frequency distribution of double haplotypes of Pfmdr-1 N86Y and Y184F in 2017-2018 samples compared with previously published reports.

\section{Supplementary Files}

This is a list of supplementary files associated with this preprint. Click to download.

- Supplementaryfile1.docx

- Supplementaryfile2.docx 